\title{
NUMERICAL SCHEME FOR ONE-PHASE 1D FRACTIONAL STEFAN PROBLEM USING THE SIMILARITY VARIABLE TECHNIQUE
}

\author{
Marek Blasik \\ Institute of Mathematics, Czestochowa University of Technology \\ Czestochowa, Poland \\ marek.blasik@im.pcz..pl
}

\begin{abstract}
In this paper we present a numerical method to solve a one-dimensional, one-phase extended Stefan problem with fractional time derivative described in the Caputo sense. The proposed method is based on applying a similarity variable for the anomalous-diffusion equation and the finite difference method. In the final part, examples of numerical results are discussed.
\end{abstract}

Keywords: Stefan problem, anomalous diffusion, fractional derivative, finite difference method

\section{Introduction}

The fractional Stefan problem (FSP) is a generalization of the classical Stefan problem. In recent years, mathematical models containing fractional differential equations were applied to model such processes as: heat transport in materials with a complex structure [1,2], controlled release of a drug from slab matrices [3-9] and movement of the shoreline in a sedimentary ocean basin [10]. Exact solutions are known for certain special models, including the case of a linear equation with constant diffusivity. Numerical schemes for the above-mentioned processes were obtained using the homotopy method. However, the comparison of the numerical and analytical solution shows that so far the developed numerical methods do not yield good results. Therefore there is a need to construct a more accurate numerical scheme.

\section{Formulation of the problem}

One-dimensional, one-phase Stefan problem describing a controlled release of a drug from slab matrices is given by subdiffusion equation

$$
{ }^{C} D_{0+, t}^{\alpha} f(x, t)=D_{\alpha} \frac{\partial^{2} f(x, t)}{\partial x^{2}}, \quad 0<x<s(t), \quad t>0
$$


supplemented with the boundary conditions

$$
f(0, t)=0, \quad f(s(t), t)=f_{m}, \quad t>0,
$$

initial conditions

$$
f(0,0)=f_{m}, \quad s(0)=0,
$$

and Stefan condition

$$
\left(f_{\max }-f_{m}\right)^{C} D_{0+, t}^{\alpha} s(t)=\left.D_{\alpha} \frac{\partial f(x, t)}{\partial x}\right|_{x=s(t)} .
$$

The operator acting on the left-hand side of equations (1) and (4) is called Caputo partial derivative of order $\alpha$ and is defined by the following formula:

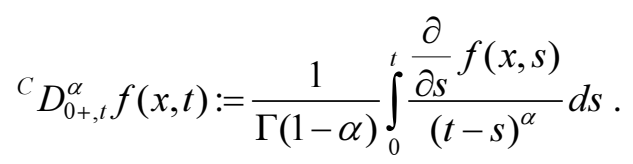

The variables and the physical parameters in equation (1) and conditions (2)-(4) denote quantities expressed in physical units, e.g. $x$ in meters, $t$ in seconds, $D_{\alpha}$ (generalized diffusivity) in square meters per second to the alpha power $\left[\mathrm{m}^{2} / \mathrm{s}^{\alpha}\right]$. We shall present the Stefan problem in non-dimensional variables. To this aim we introduce new variables:

$$
\tau=t\left(\frac{D_{\alpha}}{l^{2}}\right)^{\frac{1}{\alpha}}, \quad X=\frac{x}{l}, \quad S(\tau)=\frac{s(t)}{l}, \quad \bar{f}=\frac{f}{f_{m}},
$$

where $l$ is a certain standard length and reduces equations (1)-(4) to the following system:

$$
\begin{gathered}
{ }^{C} D_{0+, \tau}^{\alpha} \bar{f}(X, \tau)=\frac{\partial^{2} \bar{f}(X, \tau)}{\partial X^{2}}, \quad 0<X<S(\tau), \quad \tau>0, \\
\bar{f}(0, \tau)=0, \quad \bar{f}(S(\tau), \tau)=1, \quad \tau>0, \\
\bar{f}(0,0)=1, \quad s(0)=0, \\
{ }^{C} D_{0+, \tau}^{\alpha} s(\tau)=\left.\Lambda \frac{\partial \bar{f}(X, \tau)}{\partial X}\right|_{x=s(t)},
\end{gathered}
$$

where $\Lambda=\left(\frac{f_{\max }}{f_{m}}-1\right)^{-1}$ is called the Stefan number and $f_{\max }, f_{m}$ are the initial concentration of the solute distributed in the matrix and the solubility of the solute, respectively. The concentration profile is shown in Figure 1. 


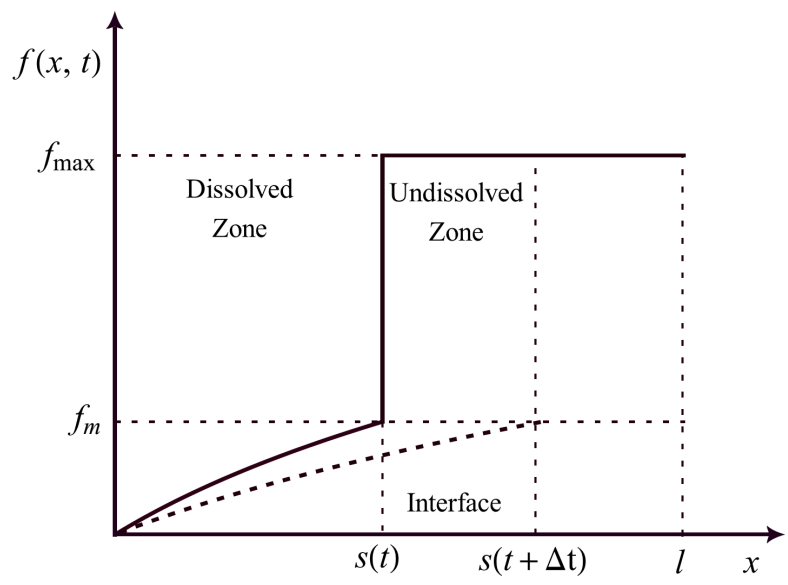

Fig. 1. Drug concentration profile [9]

\section{Solution of the problem}

The results obtained in [4] show that the movement of the dissolved/undissolved phase interface is described by power function $S(\tau)=p \tau^{\alpha / 2}$. Parameter $p$ is determined numerically from the following transcendental equation [4]:

$$
\frac{\Gamma(1+\alpha / 2)}{\Gamma(1+\alpha / 2)} p \frac{1-W(-p ;-\alpha / 2,1)}{W(-p ;-\alpha / 2,1-\alpha / 2)}=\Lambda,
$$

where Wright function $W$ is defined by the formula:

$$
W(z ; \beta, \delta):=\sum_{k=0}^{\infty} \frac{z^{k}}{k ! \Gamma(\beta k+\delta)} .
$$

Using the similarity variable:

$$
u=\frac{X}{p \tau^{\alpha / 2}}
$$

we note that position of moving boundary is known for all time instants and located at the point $u=1$. After applying the substitution (13) function $\bar{f}$ has the form:

$$
\bar{f}(X, \tau)=\bar{f}\left(\frac{X}{p \tau^{\alpha / 2}}\right)=\bar{f}(u) .
$$

Using standard mathematical manipulations associated with the conversion of the variables in the equations we obtain an integro-differential equation in the form of: 


$$
\frac{1}{\Gamma(1-\alpha)} \int_{1}^{u}\left(z^{2 / \alpha}-u^{2 / \alpha}\right)^{-\alpha} z^{2} \frac{d \bar{f}(z)}{d z} d z=\frac{1}{p^{2}} \frac{d^{2} \bar{f}(u)}{d u^{2}}
$$

supplemented with the conditions

$$
\bar{f}(0)=0, \quad \bar{f}(1)=1, \quad \bar{f}^{\prime}(1)=\Lambda^{-1} p^{2} \frac{\Gamma(1+\alpha / 2)}{\Gamma(1-\alpha / 2)} .
$$

The kernel of the integral on the left-hand side of formula (15) is difficult to use in the numerical scheme. For this reason, we apply an auxiliary variable:

$$
\bar{u}=u^{2 / \alpha}=\left(\frac{X}{p}\right)^{2 / \alpha} \frac{1}{\tau},
$$

and auxiliary function

$$
F(\bar{u})=F\left(u^{2 / \alpha}\right)=\frac{\alpha}{2} u^{3-2 / \alpha} \bar{f}^{\prime}(u) .
$$

After using the relationships (17), (18) for (15), (16) we obtain the final form of the equations:

$$
\begin{gathered}
-I_{1-, \bar{u}}^{1-\alpha} F(\bar{u})=\frac{4 u^{-1-2 \alpha}}{\alpha^{2} p^{2}}\left[\bar{u} F^{\prime}(\bar{u})+\left(1-\frac{3 \alpha}{2}\right) F(\bar{u})\right], \quad 0<\bar{u}<1, \\
F(1)=\Lambda^{-1} \frac{\alpha p^{2}}{2} \frac{\Gamma(1+\alpha / 2)}{\Gamma(1-\alpha / 2)}
\end{gathered}
$$

where the operator on the left-hand side of equation (19), called the left-sided Riemann-Liouville integral of order $1-\alpha$, is defined by the formula:

$$
I_{b-}^{\alpha} f(t):=\frac{1}{\Gamma(\alpha)} \int_{t}^{b} \frac{f(s)}{(s-t)^{1-\alpha}} d s, \quad t<b .
$$

After solving equation (19) with condition (20) we return to the original solution using formulas:

$$
\begin{gathered}
\bar{f}(X, \tau)=\bar{f}\left(\frac{X}{p \tau^{\alpha / 2}}\right)=1-\int_{u^{2 / \alpha}}^{1} \bar{u}^{-\alpha} F(\bar{u}) d \bar{u}, \\
u=\bar{u}^{\alpha / 2} .
\end{gathered}
$$




\section{Numerical method}

To solve equation (19) with condition (20), we propose the following approach based on the finite difference method. We introduce the mesh nodes:

$$
0=\bar{u}_{n+1}<\bar{u}_{n}<\bar{u}_{n-1}<\ldots<\bar{u}_{j+1}<\bar{u}_{j}<\ldots<\bar{u}_{3}<\bar{u}_{2}<\bar{u}_{1}=1,
$$

with an integer $n, h=1 / n$ and $\bar{u}_{j}=1-(j-1) h$, where $j=1, \ldots, n+1$.

We approximate the value of the derivative of auxiliary function $F$ at the point $\bar{u}_{j+1}$ by the formula:

$$
F^{\prime}\left(\bar{u}_{j+1}\right)=\frac{F\left(\bar{u}_{j}\right)-F\left(\bar{u}_{j+1}\right)}{h}=\frac{F_{j}-F_{j+1}}{h} .
$$

The left-sided Riemann-Liouville integral from formula (19) can be discretized as follows:

$$
-I_{1-, \bar{u}}^{1-\alpha} F\left(\bar{u}_{k+1}\right)=\frac{1}{\Gamma(1-\alpha)} \int_{1}^{\bar{u}_{k+1}}\left(s-\bar{u}_{k+1}\right)^{-\alpha} F(s) d s=\sum_{j=1}^{k} b_{j, k+1} F\left(\bar{u}_{j}\right),
$$

where weights

$$
b_{j, k+1}=\frac{h^{1-\alpha}}{\Gamma(2-\alpha)}\left[(k-j)^{1-\alpha}-(k+1-j)^{1-\alpha}\right],
$$

were calculated using the rectangle rule. The discrete form of the equation (19) is expressed by the formula:

$$
\sum_{j=1}^{k} b_{j, k+1} F_{j}=\frac{4 u_{k+1}^{-1-2 \alpha}}{\alpha^{2} p^{2}}\left[\bar{u}_{k+1} \frac{F_{k}-F_{k+1}}{h}+\left(1-\frac{3 \alpha}{2}\right) F_{k+1}\right],
$$

with the boundary condition:

$$
F_{1}=\Lambda^{-1} \frac{\alpha p^{2} \Gamma(1+\alpha / 2)}{2 \Gamma(1-\alpha / 2)} .
$$

From formula (28) we obtain a recursive relationship which allows us to determine the value of function $F$ at node $k+1$ using known values $F_{j}, j=1, \ldots, k$ :

$$
F_{k+1}=\frac{p^{2} \alpha^{2} \sum_{j=1}^{k} b_{j, k+1} F_{j}}{4 \bar{u}_{k+1}^{-2 \alpha}\left(\left(1-\frac{3 \alpha}{2}\right)-\frac{1}{h} \bar{u}_{k+1}\right)}-\frac{\bar{u}_{k+1} F_{k}}{h\left(1-\frac{3 \alpha}{2}\right)-\bar{u}_{k+1}} .
$$


Finally we determine the value of the function $\bar{f}$ using the auxiliary function $F$ :

$$
\bar{f}_{k}=1-h \sum_{j=1}^{k-1} \bar{u}_{j}^{-\alpha} F_{j}
$$

\section{Numerical examples}

As an example, we shall discuss solutions of equation (7) with conditions (8)-(10) for Stefan's number $\Lambda \in\{0.25,0.15,0.05\}$ and order $\alpha \in\{0.7,0.8,0.9,0.9999\}$. The simulations were performed for $h=0.001$. In Figures 2-4 concentration profiles (left side) and the movement of the dissolved/undissolved phase interface (right side) are shown. Comparisons of numerical results with approximate analytical solution are shown in Figures 5-6.
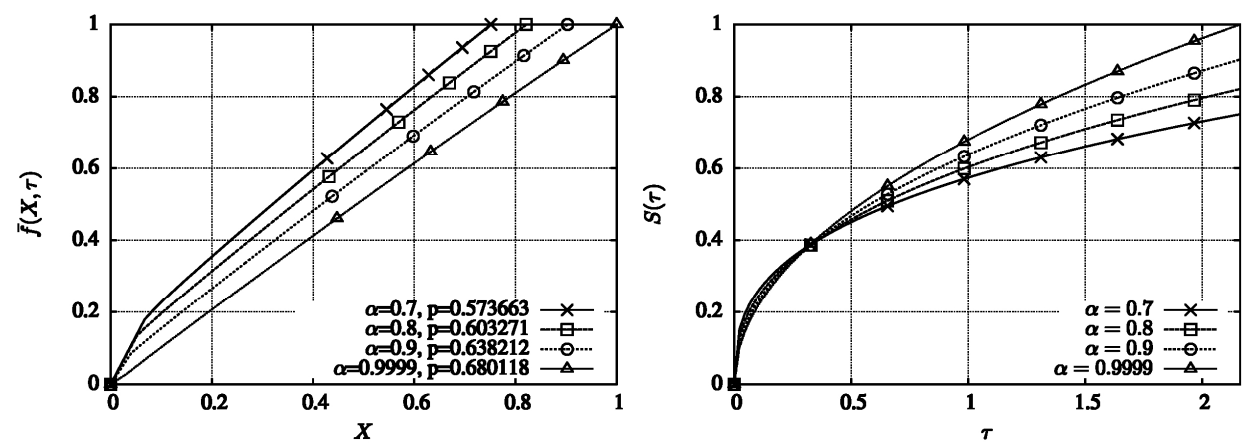

Fig. 2. Numerical solution $\bar{f}(X, \tau)$ (left side) and $S(\tau)$ (right side) for $\Lambda=0.25$ and $\tau=2.16204$
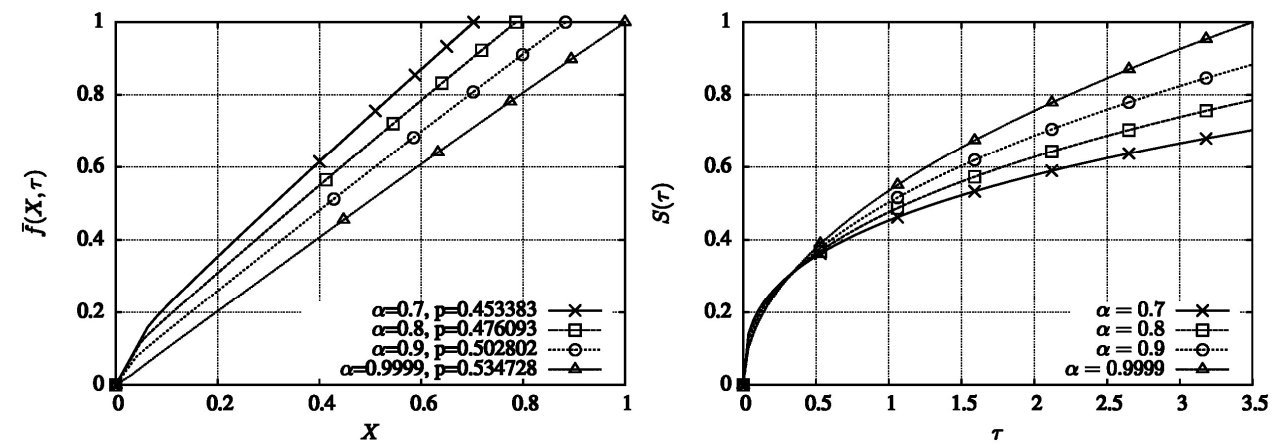

Fig. 3. Numerical solution $\bar{f}(X, \tau)$ (left side) and $S(\tau)$ (right side) for $\Lambda=0.15$ and $\tau=3.49774$ 

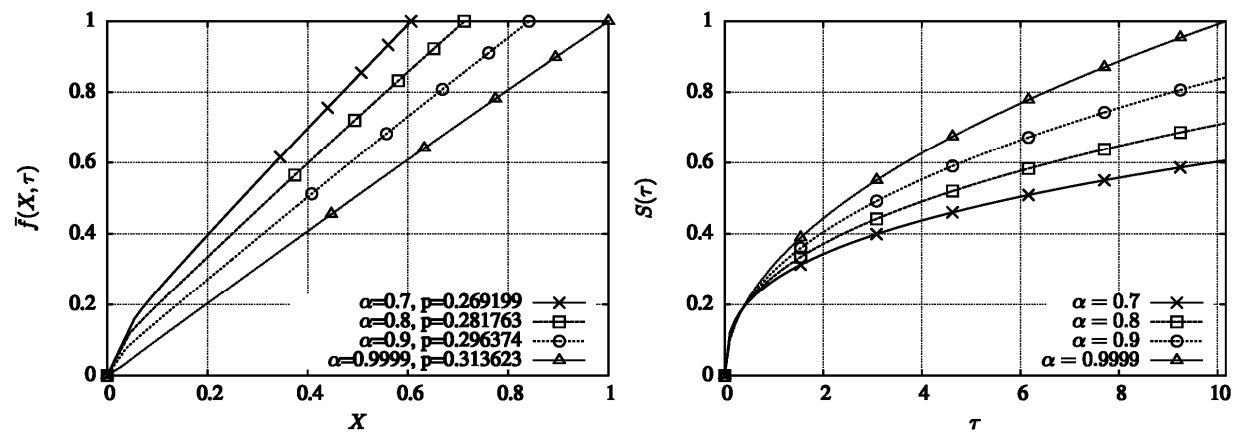

Fig. 4. Numerical solution $\bar{f}(X, \tau)$ (left side) and $S(\tau)$ (right side) for $\Lambda=0.05$ and $\tau=10.1692$
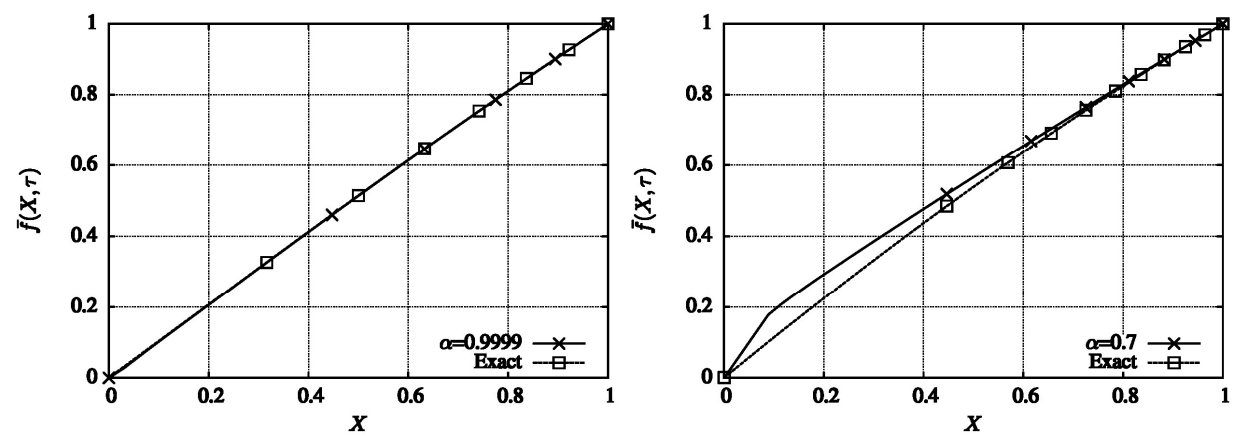

Fig. 5. Comparison of numerical solution with approximate analytical solution for $\Lambda=0.25$ and $\tau=2.16204$
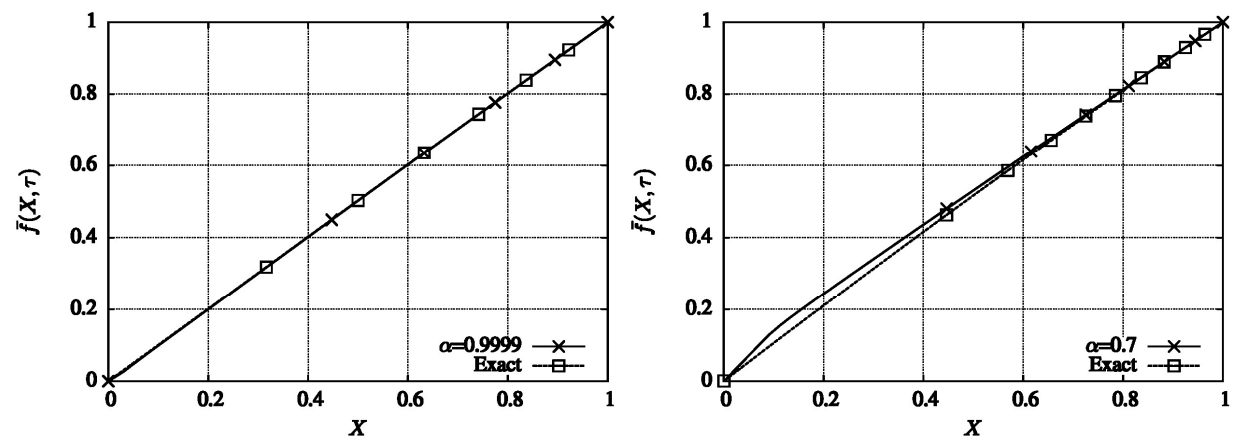

Fig. 6. Comparison of numerical solution with approximate analytical solution for $\Lambda=0.05$ and $\tau=10.1692$

\section{Conclusions}

In the paper we constructed a numerical method for solving the Stefan problem in anomalous diffusion. This problem is here formulated so as to describe the solute transfer which does not obey the classical diffusion equation with classical 
Stefan condition $[3,7,8]$. This and other practical applications of the fractional calculus approach to moving boundary problems $[1-3,7,8]$ indicate that there is a need to develop numerical methods to determine the solution and characteristics of the process such as the value of parameter $p$, defining the movement of the phase interface.

Comparing the numerical results presented in Figures 2-4, we observe that the complete dissolution time depends on the fractional order $\alpha$ (of the Caputo derivative w.r.t. to the time) and on the value of Stefan number $\Lambda$. First, the process slows down when order $\alpha$ decreases. Thus, the fractional Stefan problem describes process delayed in comparison to the classical model. Next, Stefan number $\Lambda$ is related to solubility $f_{m}$. When solubility is reduced, then $\Lambda$ also decreases and the complete dissolution is delayed. Such behavior is analogous both in the classical and anomalous case.

Let us note that the exact solutions for the Stefan problem with constant diffusivity/solubility are known for the classical and anomalous problems. Hence the outcome of the constructed numerical scheme can be compared to the exact results. In this way we are able to predict when our numerical approach, using similarity variable technique and reduced equation, will be most useful.

We illustrate the error of the numerical scheme for two examples of fractional order and two different values of the Stefan number. In Figures 5-6 the numerical and exact solution graphs are drawn for the complete dissolution time. The proposed numerical scheme works very well for order of Caputo derivative close to the classical value $\alpha=1$. When order of Caputo derivative decreases, then the scheme generates higher error as $X$ tends to 0 . Similarly, the value of Stefan number $\Lambda$ affects the accuracy of the numerical results. For lower value of $\Lambda$ we obtain a better consistency with analytic results. In all cases the error increases in the neighbourhood of $X=0$.

In conclusion, the numerical method produces more accurate results for low values of Stefan number and fractional order close to the classical value. The further work will include an extended accuracy/stability analysis of the developed method and the new construction, also based on the similarity variable but applying the front-fixing approach. This method, known in classical moving boundary problems but new in fractional numerical analysis, will be the subject of our subsequent paper.

\section{Acknowledgments}

This work was supported by the Czestochowa University of Technology Grant Number BS/MN-1-105-301/13/P.

\section{References}

[1] Aoki Y., Sen M., Paolucci S., Approximation of transient temperatures in complex geometries using fractional derivatives, Heat Mass Transfer 2008, 44, 771-777.

[2] Voller V., An exact solution of a limit case Stefan problem governed by a fractional diffusion equation, International Journal of Heat and Mass Transfer 2010, 53, 5622-5625. 
[3] Liu Junyi, Xu Mingyu, An exact solution to the moving boundary problem with fractional anomalous diffusion in drug release devices, Zeitschrift für Angewandte Mathematik und Mechanik 2004, 84, 22-28.

[4] Liu Junyi, Xu Mingyu, Some exact solutions to Stefan problems with fractional differential equations, Journal of Mathematical Analysis and Applications 2009, 351, 536-542.

[5] Xicheng Li, Mingyu Xu, Xiaoyun Jiang, Homotopy perturbation method to time-fractional diffusion equation with a moving boundary condition, Applied Mathematics and Computation 2009, 208, 434-439.

[6] Xicheng Li, Mingyu Xu, Shaowei Wang, Scale-invariant solutions to partial differential equations of fractional order with a moving boundary condition, Journal of Physics A: Mathematical and Theoretical 2008, 41, 155-202.

[7] Li Xi-cheng, Fractional Moving Boundary Problems and Some of Its Applications to Controlled Release System of Drug, PhD thesis, Shandong University 2009.

[8] Chen Yin, Xicheng Li, Anomalous diffusion of drug release from slab matrix: Fractional diffusion models, International Journal of Pharmaceutics 2011, 418, 78-87.

[9] Błasik M., Klimek M., Numerical scheme for the one-phase 1D fractional Stefan problem, Proceedings of the 20th CMM 2013 International Conference on Computer Methods in Mechanics, Poznań, Poland, 27-31 August 2013.

[10] Rajeev, Kushwaha M.S., Homotopy perturbation method for a limit case Stefan problem governed by fractional diffusion equation, Applied Mathematical Modelling 2013, 37, 3589-3599. 
